\title{
AVALIAÇÃO DO NÍVEL DE CONHECIMENTO SOBRE POLÍTICA DE SAÚDE COMO PREDITOR DE DESENVOLVIMENTO REGIONAL
}

\author{
Aline Xavier dos Santos Germano ${ }^{1}$ \\ Centro Federal de Educação Tecnólogica Celso Suckow da Fonseca \\ aline.santos@cefet-rj.br \\ Ericson de Oliveira Paes Leme ${ }^{2}$ \\ Centro Federal de Educação Tecnólogica Celso Suckow da Fonseca \\ ericson.leme@aluno.cefet-rj.br \\ Tatiana de Souza ${ }^{3}$ \\ Centro Federal de Educação Tecnólogica Celso Suckow da Fonseca \\ pandoramc@yahoo.com.br \\ Cristiane Rosa Magalhães ${ }^{4}$ \\ Centro Federal de Educação Tecnólogica Celso Suckow da Fonseca \\ cristiane.magalhaes@cefet-rj.br \\ Júlio Cesar Santos da Silva ${ }^{5}$ \\ Centro Federal de Educação Tecnólogica Celso Suckow da Fonseca \\ julio.silva@cefet-rj.br
}

\begin{abstract}
Resumo
Este artigo tem como objetivo o levantamento do conhecimento da população em geral e pelos profissionais da área de saúde, sobre o Sistema Único de Saúde brasileiro, seus campos de atuação e ações praticadas. Tendo em vista a influência e importância do mesmo para o desenvolvimento regional. Inicialmente foi realizada uma breve exposição sobre esses fatores de conhecimentos citados e uma pesquisa de levantamento de natureza aplicada e objetivo descritivo com delineamento transversal, para mensurar o nível de conhecimento do público-alvo. A pesquisa demonstrou que o nível de conhecimento da população sobre o assunto está abaixo do esperado, considerando a relevância do SUS, sua participação social e contribuição para o desenvolvimento regional.
\end{abstract}

Palavras-chave: Sistema Único de Saúde (SUS). Desenvolvimento Regional. Políticas de Saúde.

\section{EVALUATION OF THE LEVEL OF KNOWLEDGE ABOUT HEALTH POLICY AS PREDICTOR OF REGIONAL DEVELOPMENT}

\begin{abstract}
This article aims to survey the knowledge of the population in general and by health professionals, about the Brazilian Unified Health System, its fields of action and actions taken. In view of its influence and importance for regional development. Initially, a brief presentation was made on these knowledge factors mentioned and a survey survey of an applied nature and descriptive objective with a cross-sectional design, to measure the level of knowledge of the target audience. The research showed that the population's level of knowledge on the subject is below expectations, considering the relevance of SUS, its social participation and contribution to regional development.
\end{abstract}

Keywords: Unified Health System (SUS). Regional Development. Health Policies.

\footnotetext{
${ }^{1}$ Mestranda em Desenvolvimento Regional e Sistemas Produtivos (PPDSP). https://orcid.org/0000-0002-8081-9379.

${ }^{2}$ Mestrando em Desenvolvimento Regional e Sistemas Produtivos (PPDSP). https://orcid.org/0000-0002-6815-3244.

${ }^{3}$ Mestranda em Desenvolvimento Regional e Sistemas Produtivos (PPDSP). https://orcid.org/0000-0002-4290-5591.

${ }^{4}$ Doutora (Neurobiologia) - Instituto de Biologia da Universidade Federal Fluminense. https://orcid.org/0000-0002-7231-3431.

${ }^{5}$ Doutor em Enfermagem Médico curúrgica pela Universidade Federal do Rio de Janeiro. http://orcid.org/0000-0001-7223-
} 3717. 


\section{ARTIGO}

INOVAÇÃo

\section{INTRODUÇÃO}

O Sistema Único de Saúde (SUS) brasileiro está entre os maiores sistemas de saúde do mundo e engloba diversas atividades, que variam das mais simples, relacionadas à atenção primária à saúde, até as atividades de alta complexidade como transplante de órgãos. "O SUS foi um projeto que assumiu e consagrou os princípios da Universalidade, Equidade e Integralidade da atenção à saúde da população brasileira" (TEIXEIRA; CRUZ; AZEVEDO, 2016).

Segundo Passos (2018), a saúde deixou de ser referência apenas para cura e ausência de doenças e passou a ter foco também na promoção à saúde, prevenção de doenças, tratamento e reabilitação. A partir da Constituição de 1988, a saúde foi instituída como direito de todos e dever do Estado e teve seus princípios e diretrizes estabelecidos pela lei orgânica 8080/90 e pela lei 8142/90. De acordo com a lei no 8.080 de 19 de setembro de 1990, “o SUS é constituído pelo conjunto de ações e serviços de saúde, prestados por órgãos e instituições públicas federais, estaduais e municipais, da Administração direta e indireta e das fundações mantidas pelo Poder Público." A evolução do sistema brasileiro de saúde caminhou juntamente com os setores políticos e econômicos do país. Inicialmente a assistência à saúde era focada nos momentos de endemias e pandemias, pois afetavam diretamente o setor econômico e social ameaçando o modelo capitalista adotado à época. (AGUIAR, 2015).

Diversos campos de atuação são abrangidos pelo SUS, entre eles estão a execução de ações de vigilância sanitária, epidemiológica, de saúde do trabalhador e de assistência terapêutica integral, inclusive farmacêutica, a participação na formulação da política e na execução de ações de saneamento básico, a ordenação da formação de recursos humanos na área de saúde, a vigilância nutricional e a orientação alimentar, a colaboração na proteção do meio ambiente, nele compreendido o do trabalho, a formulação da política de medicamentos, equipamentos, imunobiológicos e outros insumos de interesse para a saúde e a participação, o controle e a fiscalização de serviços, produtos e substâncias de interesse para a saúde, a fiscalização e a inspeção de alimentos, água e bebidas para consumo humano, a participação no controle e na fiscalização da produção, transporte, guarda e utilização de substâncias e produtos psicoativos, tóxicos e radioativos, o incremento, em sua área de atuação, do desenvolvimento científico e tecnológico, a formulação e execução da política de sangue e seus derivados.

Atualmente, o setor saúde tem sido considerado como inquestionável indutor e parte constitutiva do modelo de desenvolvimento (GADELHA, 2007). Considerando que, a saúde é um dos fatores para a melhoria da qualidade de vida da população, entende-se que a Política 
Nacional de Desenvolvimento Regional (PNDR), atualizada pelo Decreto 9.810/2019, é transversal a este estudo, tendo em vista que esta Política tem entre seus quatro objetivos prioritários: I - Promover a convergência dos níveis de desenvolvimento e de qualidade de vida inter e intra regiões brasileiras e a equidade no acesso a oportunidades de desenvolvimento em regiões que apresentem baixos indicadores socioeconômicos (BRASIL, 2019). Entendendo que as peculiaridades do complexo da saúde, relacionadas ao seu dinamismo, o elevado grau de inovação e potencial de geração de renda e emprego o transformam em um conjunto articulado de atividades econômicas que impactam sensivelmente no desenvolvimento econômico e social (GADELHA, 2007). Na articulação com vertente desenvolvimentista, a saúde configura-se como uma das principais áreas a serem planejadas e implementadas, seja pela necessidade de abordagem e planejamento regional da saúde, seja porque fronteiras epidemiológicas não se restringem a delimitações políticas e institucionais (GADELHA, 2007).

A área da saúde em toda a sua amplitude possui um papel de destaque no desenvolvimento. Por um lado, constitui-se um importante e vital vetor de integração entre a sociedade e os cidadãos, possuindo um papel estratégico para a PNDR (GADELHA, 2007). Os desafios são imensos, mas este talvez possa se constituir num dos grandes marcos para a consolidação de uma visão não setorial da saúde, evidenciando sua relação com as condições de vida dos cidadãos brasileiros (GADELHA, 2007).

Sendo assim, fazendo-se uma análise do contexto do SUS, sob a ótica da PNDR, tornase visível uma crescente preocupação com o desenvolvimento regional tem marcado o contexto das políticas públicas no Brasil, sobretudo, no entendimento que o desenvolvimento regional não se restringe a matriz econômica, mais também na qualidade de vida e saúde da população.

Neste sentido, torna-se necessário o levantamento acerca do conhecimento da população sobre o SUS. Considerando a participação social no SUS através das Conferências de Saúde e dos Conselhos de Saúde na esfera municipal, estadual e federal, nos questionamos o quanto a população conhece o SUS? Sabem quais são as ações que o SUS está inserido? E qual é a opinião sobre os serviços de saúde? Para dar resposta à estes questionamentos, foi estabelecido como objetivos deste estudo analisar o nível de conhecimento acerca do SUS ,pela população brasileira e especialmente pelos profissionais de saúde, investigar quais ações ligadas a saúde são reconhecidas como ações praticadas pelo SUS, tanto pela população em geral, quanto pelos trabalhadores da saúde, avaliando também o perfil dos brasileiros que possuem este conhecimento e identificar quais as contribuições deste conhecimento para o desenvolvimento regional. 


\section{ARTIGO}

INOVAÇÃo

\section{METODOLOGIA}

Trata-se de uma pesquisa de levantamento de natureza aplicada e objetivo descritivo com delineamento transversal. Os sujeitos da pesquisa foram 252 indivíduos, de ambos os sexos, a partir de 15 anos de idade, que estavam em distanciamento social, durante a pandemia COVID-19, recomendado pela OMS e autoridades de saúde locais e que aceitaram participar da pesquisa e quando menores de idade tiveram o assentimento do responsável legal.

A amostra investigada foi não probabilística e a técnica utilizada foi a bola de neve (snowball). Tendo em vista que se trata de processo permanente de coleta de informações, que busca tirar proveito das redes sociais dos entrevistados identificados para fornecer ao pesquisador com um conjunto cada vez maior de contatos potenciais, sendo que o processo pode ser finalizado a partir do critério de ponto de saturação (VINUTO, 2014).

Os participantes foram membros da comunidade de um Centro Federal de Educação Tecnológica na região Metropolitana do Rio de Janeiro, configurando por tanto uma por amostra por conveniência.

O link para acesso ao questionário foi divulgado através de um aplicativo multiplataforma de mensagens instantâneas e de uma mídia e rede social virtual e a geração de dados ocorreu no mês de abril de 2020. Para a coleta de dados foi utilizado o questionário virtual elaborado através de um serviço gratuito para criação formulários online.

Este instrumento é composto por 12 perguntas fechadas. As perguntas iniciais são sobre dados pessoais como idade, gênero, grau de escolaridade e as demais são direcionadas a pesquisa propriamente dita. Por se tratar de uma pesquisa virtual, foi criada uma secção inicial de preenchimento obrigatório com orientações, o arquivo do consentimento livre e esclarecido (TCLE) e termo de assentimento para download e campo para manifestação seu aceite ou não em participar. Havendo, não concordância em participar da pesquisa, uma mensagem de agradecimento é feita ao participante.

Quanto à análise, os dados gerados sofreram contabilização conforme as categorias elaboradas e análise dos dados quantitativos, foi realizada com o auxílio dos softwares Atlas TI e EPI INFO e apresentados em gráficos e/ou tabelas e discutidos sobre a luz dos principais referenciais atinentes ao assunto.

Este estudo é executado em paralelo as atividades da Disciplina de Saúde pública do Mestrado em desenvolvimento regional e sistemas produtivos do Centro Federal de Educação Tecnológica Celso Suckow da Fonseca - CEFET/RJ Unidade Descentralizada de Nova Iguaçu 
e vinculado ao Grupo de pesquisas: Estudos de prevenção de riscos e agravos à saúde encontrase registrado no Diretório dos Grupos de Pesquisa do Brasil (DGP) -CNPq/Lattes.

A pesquisa central foi autorizada pelo Comitê de Ética em Pesquisa CAAE: 53984716.0.0000.5254 Parecer: 1.615.195 e respeita os princípios da autonomia, nãomaleficência, beneficência, justiça e equidade, conforme prevê a Resolução 466/2012 do Conselho Nacional de Saúde, que dispõe sobre pesquisa envolvendo seres humanos (BRASIL, 2012).

\section{RESULTADOS: perfil dos participantes}

Quanto ao sexo: a maioria dos participantes é do sexo feminino, cerca de 77,4\% como podemos ver no gráfico abaixo:

Gráfico 1: Sexo

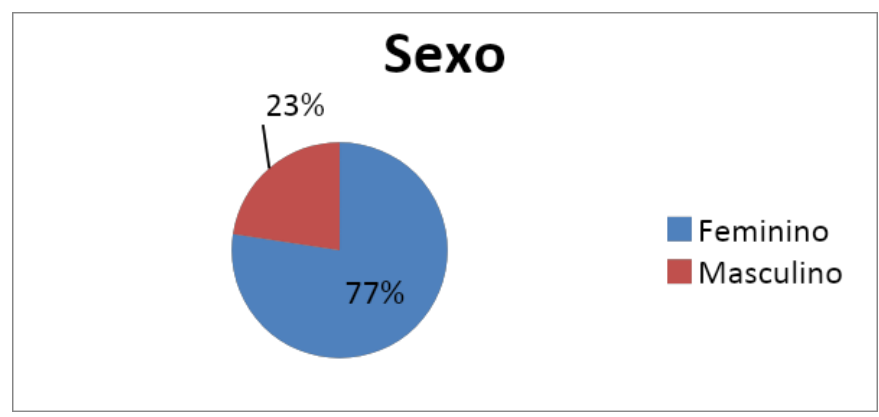

Fonte: Elaborado pelos autores.

Quanto a faixa etária: a maioria dos participantes se encontra na faixa de 36 a 45 anos, por volta de $36,5 \%$ dos participantes e mais de 50\% está compreendido entre 26 e 45 anos de idade como ilustra o gráfico abaixo:

Gráfico 2: Faixa etária

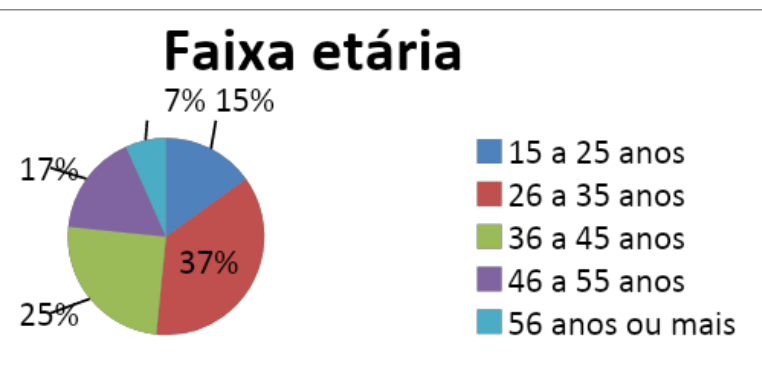

Fonte: Elaborado pelos autores. 


\section{ARTIGO}

INOVAÇÃo

Quanto ao nível de escolaridade: a maioria, cerca de 48,4\%, possui pós-graduação e 80,5\% dos participantes possuem pelo menos graduação de nível superior, conforme o gráfico 3:

Gráfico 3: Nível de escolaridade

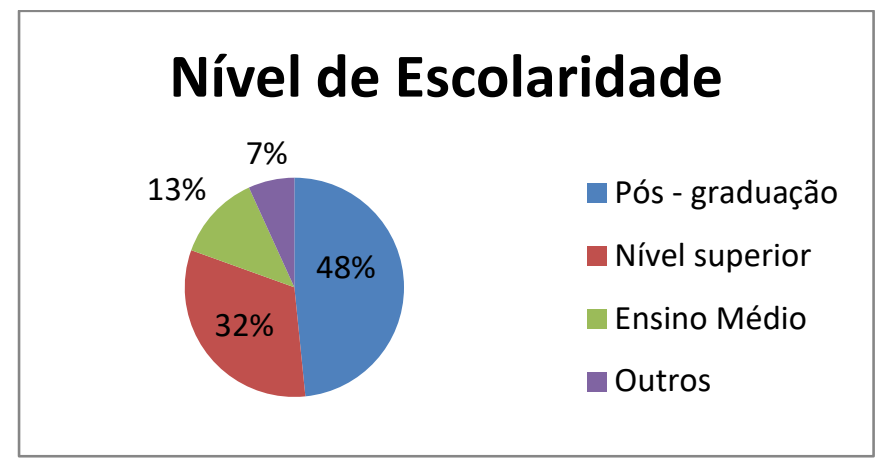

Fonte: Elaborado pelos autores.

Quanto a área profissional: a pesquisa tem como um dos objetivos fazer a apuração e comparação entre o conhecimento sobre o SUS pelos profissionais da área de saúde e os demais participantes. Dentre os participantes 16,7\% são da área da saúde e 83,3\% são de outras áreas (gráfico 4).

Gráfico 4: Área Profissional

\section{Área Profissional}

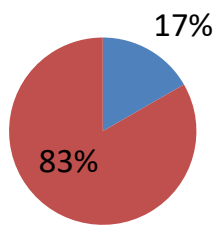

- Área da saúde

- Outras

Fonte: Elaborado pelos autores.

\section{RESULTADOS: CONHECIMENTO SOBRE AS ÁREAS ABRANGIDAS PELO SUS E AVALIAÇÃO DA QUALIDADE DO SERVIÇO PRESTADO}

A maioria dos entrevistados, que inclui 92,5\% dos participantes diz já ter utilizado o SUS. A avaliação dos mesmos sobre a qualidade do serviço é representada pelo gráfico abaixo, onde $42,6 \%$ dos entrevistados classifica o serviço como regular o que representa a maioria das 
opiniões, seguido por $32,2 \%$ do grupo que classifica o serviço como bom e $14 \%$ como muito bom. Apenas $2 \%$ identificam uma excelência no serviço prestado e $9 \%$ como péssimo, conforme o gráfico 5 :

Gráfico 5: Avaliação do serviço prestado

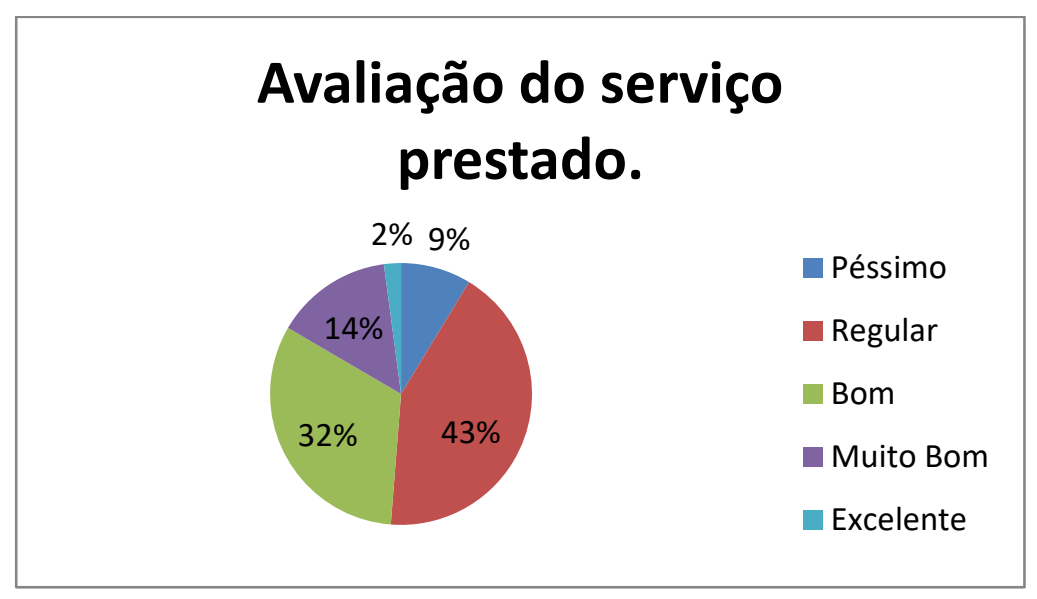

Fonte: Elaborado pelos autores.

Um pouco mais que a metade dos participantes possuem plano de saúde cerca de 54,8\% e grande parte dos entrevistados $87,4 \%$, que não possuem plano de saúde gostariam de ter. Boa parte do grupo, formada por $64,3 \%$ dizem buscar algum atendimento no SUS, porém mais que a metade destes por volta de 53,8\% somente em caso de emergência. Praticamente todos os entrevistados, 98,8\% dizem já ter se vacinado em postos de saúde públicos, enquanto apenas $1,2 \%$ dizem ter se vacinado apenas na rede particular.

Sobre as ações que os participantes acreditam serem praticadas pelo SUS, podemos ver na tabela 1 o percentual de participantes que acreditam que a ação citada é praticada pelo SUS. A tabela apresenta os dados do público geral, e, também do grupo formado apenas pelos profissionais da saúde entrevistados. 
Tabela 1: Percentual de participantes que reconhecem a ação citada como uma ação praticada pelo SUS.

\begin{tabular}{|c|c|c|}
\hline Ações Praticadas pelo SUS & Público Geral & Profissionais da Saúde \\
\hline Vigilância Sanitária. & $45,20 \%$ & $74,41 \%$ \\
\hline Vigilância Epidemiológica. & $76,60 \%$ & $81,39 \%$ \\
\hline Saúde do trabalhador. & $68,30 \%$ & $86,04 \%$ \\
\hline Assistência terapêutica integral, inclusive farmacêutica. & $66,30 \%$ & $83,72 \%$ \\
\hline $\begin{array}{l}\text { A ordenação da formação de recursos humanos na } \\
\text { área de saúde. }\end{array}$ & $43,30 \%$ & $65,11 \%$ \\
\hline Vigilância nutricional e a orientação alimentar. & $56,30 \%$ & $72,09 \%$ \\
\hline $\begin{array}{l}\text { Colaboração na proteção do meio ambiente, nele } \\
\text { compreendido o do trabalho. }\end{array}$ & $27 \%$ & $58,13 \%$ \\
\hline $\begin{array}{l}\text { Formulação da política de medicamentos, } \\
\text { equipamentos, imunobiológicos e outros insumos de } \\
\text { interesse para a saúde e a participação na sua } \\
\text { produção. }\end{array}$ & $63,50 \%$ & $81,39 \%$ \\
\hline $\begin{array}{l}\text { Controle e a fiscalização de serviços, produtos e } \\
\text { substâncias de interesse para a saúde; }\end{array}$ & $59,50 \%$ & $81,39 \%$ \\
\hline $\begin{array}{l}\text { Fiscalização e a inspeção de alimentos, água e bebidas } \\
\text { para consumo humano. }\end{array}$ & $28,20 \%$ & $58,13 \%$ \\
\hline $\begin{array}{l}\text { Participação no controle e na fiscalização da produção, } \\
\text { transporte, guarda e utilização de substâncias e } \\
\text { produtos psicoativos, tóxicos e radioativos. }\end{array}$ & $31,70 \%$ & $51,16 \%$ \\
\hline $\begin{array}{l}\text { O incremento, em sua área de atuação, do } \\
\text { desenvolvimento científico e tecnológico. }\end{array}$ & $37,30 \%$ & $67,44 \%$ \\
\hline $\begin{array}{l}\text { Formulação e execução da política de sangue e seus } \\
\text { derivados. }\end{array}$ & $52,40 \%$ & $62,79 \%$ \\
\hline
\end{tabular}

Fonte: Elaborado pelos autores.

Considerando o público geral a ação mais conhecida é a de "Vigilância epidemiológica", já entre os profissionais da saúde é a de "Saúde do trabalhador". A atividade menos conhecida entre o público geral é a de "Colaboração na proteção do meio ambiente, nele compreendido o do trabalho.”, já entre os profissionais da saúde é a de "Participação no controle e na fiscalização da produção, transporte, guarda e utilização de substâncias e produtos psicoativos, tóxicos e radioativos.”. Outro dado relevante é que menos da metade dos profissionais da saúde reconhecem todas as atividades praticadas pelo SUS, um total de $41,86 \%$, mas o grupo tem a maior conhecimento sobre todas as atividades, se compararmos ao público geral. 


\section{DISCUSSÃO}

A Política de Promoção da Saúde, destacando a promoção da saúde como uma estratégia de possibilidade de enfocar os aspectos que determinam o processo saúde-adoecimento, como a violência, desemprego, subemprego, falta de saneamento básico, habitação inadequada e/ou ausente, dificuldade de acesso à educação, fome, urbanização desordenada, qualidade do ar e da água ameaçada e deteriorada; e potencializa formas amplas de intervir em saúde (BRASIL, 2010b).

Na compreensão de que a Constituição Federal Brasileira (CFB), de 1988, determina que a saúde é um direito de todos e um dever do Estado. Torna-se necessário o desenvolvimento de ações visando o desenvolvimento de mecanismos e ferramentas de legitimação dos SUS. A política de saúde instituiu um modelo de atenção universal e de controle único de todos os serviços públicos e privados, o SUS, a partir do qual se tornou possível a organização dos diferentes níveis de atenção (SACRAMENTO, 2016).

As políticas sociais em geral, e a política de saúde em particular, representam historicamente um avanço fundamental nas relações sociais das sociedades capitalistas (SACRAMENTO, 2016). A ação das políticas públicas repercute na economia e na sociedade, o que caracteriza que qualquer teoria sobre o tema, necessita explicar as inter-relações entre Estado, política, economia e sociedade (SANTOS, 2016).

Apesar de terem acontecidos diversos avanços no contexto social e de saúde, é imperativo destacar que a articulação da prestação dos serviços do SUS envolve uma interatividade central dos complexos tecnológicos e industriais da produção de medicamentos, vacinas, equipamentos médicos, insumos para diagnóstico e hemoderivados (SACRAMENTO, 2016). Consideramos que no Brasil, a cobertura universal em saúde seria uma forma de ampliar os espaços, evidencia-se que a efetivação da universalização dos serviços em saúde tem enfrentado uma série de obstáculos com as restrições financeiras, particularmente em relação à cobertura e oferta de serviços e gerência administrativa (SACRAMENTO, 2016)

A saúde pública baseia-se, então, no princípio do acesso universal e igualitário. O acesso igualitário significa que pessoas em uma mesma situação clínica deverão receber idêntico atendimento, ou seja, não podem ocorrer privilégios na realização dos serviços na área da saúde, a não ser aqueles relativos a necessidades clínicas (SANTOS, 2016). Sendo assim, a promoção de saúde tem o intuito de impulsionar a qualidade de vida e reduzir vulnerabilidade e riscos à saúde, relacionados aos seus determinantes e condicionantes, entre eles, o ambiente (SANTOS 2016) 
Nesta linha de raciocínio, torna-se evidente que diversas ações do SUS, irão contribuir para o desenvolvimento das ações de saúde. A participação na formulação da política e na execução de ações de saneamento básico que está intimamente ligado a questão da saúde pública, tanto no que tange a promoção a saúde quanto na prevenção de doenças. Podendo-se dizer que o saneamento orientado para a Promoção da Saúde abrange a implantação de uma estrutura física composta por sistemas de engenharia, o que o caracteriza. O saneamento constitui uma intervenção de engenharia que ocorre no ambiente considerado como espaço físico, voltada para obstaculizar a transmissão de doenças e assegurar a salubridade ambiental, e que compreende a saúde como ausência de doenças (SOUZA e FREITAS. 2010).

A Fundação Nacional de Saúde a (FUNASA), está ligada a implementação do SUS e promove o fortalecimento das instituições estaduais e municipais utilizando recursos que desoneram as tarifas dos serviços e aceleram a universalização do atendimento dos serviços. A Funasa atualmente promove o fomento às soluções de saneamento para prevenção e controle de doenças.

Estratégias diversas são estabelecidas, sobretudo, no contexto cotidiano, para a prevenção de riscos e agravos à saúde, dentre as diversas circunstâncias possíveis de prevenção e realizadas pelo SUS, está a vigilância nutricional e orientação alimentar. A má alimentação é um dos principais fatores de risco relacionados à carga global de doenças no mundo. No Brasil, em 2015, ela foi o fator de risco que mais contribuiu para os anos de vida perdidos, sendo superior, inclusive, ao uso de álcool, drogas, tabagismo e inatividade física. Ainda, a alimentação inadequada foi o principal fator de risco para mortes no mundo em 2017. (BORTOLINI, OLIVEIRA, SILVA, SANTIN, MEDEIROS, SPANIOL, PIRES, ALVES E FALLER, 2020).

Podemos encontrar o programa de vigilância alimentar e nutricional nos serviços de saúde no site do Ministério da saúde, na responsabilidade da Secretaria de Atenção Primária a Saúde. O programa inclui a avaliação antropométrica (medição de peso e estatura) e do consumo alimentar, esses dados são inseridos no Sistema de Vigilância Alimentar e Nutricional (SISVAN), para o apoio de gestores e profissionais de saúde no processo de organização e avaliação da atenção nutricional.

A vertente de formação de recursos humanos para a área da saúde, segundo o site do Ministério da saúde, a secretaria de Gestão do Trabalho e da Educação na Saúde (SGTES) atualmente é responsável por formular políticas públicas orientadoras da gestão, formação e qualificação dos trabalhadores e da regulação profissional na área da saúde no Brasil. A SGTES tem a missão de promover a integração dos setores de saúde e educação e de administrar a 
INOVAÇÃo

relação entre as gestões federal, estaduais e municipais do SUS, incluindo qualificação profissional e distribuição das ofertas de trabalho na área da saúde.

Baseado na Política de Educação e Desenvolvimento do SUS, foram criadas abordagens permanentes de educação em saúde. A política fortalece centros permanentes de educação em saúde, que por sua vez incentivaram a revisão da educação em saúde, assistência, estratégias de gestão, formulação de políticas e controle social. Com base em instituições de educação permanente como uma de suas diretrizes, o departamento realizou o papel de qualificar profissionais e trabalhadores da saúde no Brasil (AMARAL, CAVALCANTE, FARIAS, RIBEIRO, JÚNIOR E GOMES, 2018).

Também é discutido no âmbito do SUS a formulação da política de medicamentos, equipamentos, imunobiológicos e outros insumos de interesse para a saúde. O acesso a medicamentos, equipamentos e imunobiológicos de qualidade, são fundamentais para a manutenção da saúde da população. O Ministério da saúde promove um conjunto de ações para promoção, proteção e recuperação da saúde viabilizando o acesso e uso racional de medicamentos. Segundo informações do Ministério da saúde, o suprimento regular de imunobiológicos de qualidade comprovada e os quantitativos necessários para atendimento à população são a principal garantia para a prevenção das doenças imunopreveníveis e para o tratamento com os soros.

$\mathrm{O}$ uso racional de medicamentos tem sido apontado, juntamente com o acesso a medicamentos de qualidade, como objetivo central de uma política de medicamentos. A partir do marco da Conferência de Nairobi, se reconheceu que os benefícios do acesso não se concretizam, ou mesmo perdem, se os medicamentos não forem usados de forma adequada." (BERMUDEZ, ESHER, OSORIO-DE-CASTRO, VASCONCELOS, CHAVES, OLIVEIRA, SILVA E LUIZA, 2018).

O campo de atuação do SUS é amplo e abrange a Vigilância Epidemiológica, a Vigilância Sanitária, a Vigilância Nutricional além da saúde do trabalhador. A Vigilância Sanitária tem um caráter prioritário porque é entendida como uma ação preventiva de acordo com a diretriz do SUS sobre integralidade do atendimento com prioridade para prevenção. (LUCCHESE, 2008).

Os serviços de interesse para saúde podem estar no contexto hospitalar e também fora dele tais como: salões de beleza, clínicas de estética, estúdios de tatuagem, academias de ginástica, creches e asilos (ANVISA, 2020).

Os produtos e substâncias são equipamentos de uso ou aplicação médica, hospitalar odontológica ou laboratorial, os cosméticos (hidratantes, maquiagem, alisantes capilares, etc), 
perfumes, produtos de higiene pessoal (xampu, sabonete, creme dental, etc), saneantes domissanitários (detergentes, desinfetantes, inseticidas, etc). (SÃO PAULO, 2019).

A participação da sociedade na vigilância sanitária confere um espaço de exercício da cidadania quando inclui a população por meio dos conselhos de saúde, na fiscalização e na tomada de decisão sobre os produtos e serviços de interesse coletivo reforçando assim a dinâmica social (CRUZ et al. 2012)

As atividades de fiscalização e de inspeção de produtos (alimentos, água e bebidas) para consumo humano englobam complexidade e dinamismo que exigem integração e articulação entre o Estado e a sociedade. Devido ao imensurável risco na produção, manipulação e disponibilização desses produtos a Vigilância Sanitária juntamente com a participação social possuem grande responsabilidade nas práticas sanitárias (COSTA et ROZENFELD, 2000).

No tocante a ação do SUS no controle e na fiscalização da produção, transporte e guarda e utilização de substâncias e produtos psicoativos, tóxicos e radioativos. Cabe a Vigilância Sanitária fiscalizar e gerenciar os riscos de exposição, produção, transporte, guarda e utilização das substâncias com potencial de causar danos à saúde humana. Os produtos potencialmente perigosos são definidos como: "todo aquele que represente risco à saúde das pessoas, ao meio ambiente ou à segurança pública, seja ele encontrado na natureza ou produzido por qualquer processo." (BATAEIRO, 2016)

Quanto ao incremento, em sua área de atuação, do desenvolvimento científico e tecnológico, evidenciou-se a criação da política de inovação a partir da I Conferência Nacional de Ciência e Tecnologia em Saúde, culminando na Política Nacional de Ciência, Tecnologia e Inovação na Saúde com o objetivo de compartilhar e fomentar pesquisas e avanços alcançados na área da saúde.

A crescente demanda sobre temas que envolvem a saúde humana exigem cada vez mais inovações, pesquisas científicas e aparatos tecnológicos à medida que medicamentos, vacinas e diagnósticos também seguem em ascenção (FIOCRUZ, 2020).

Ainda deve ser destacada a formulação e execução da política de sangue e seus derivados, visto que a Organização Mundial da Saúde estabelece que o sangue e seus derivados são artigos de alta vigilância. Na Constituição Federal Brasileira têm-se como "sangue, componentes e seus derivados os produtos e os subprodutos originados do sangue humano venoso, placentário ou de cordão umbilical indicados para diagnóstico, prevenção e tratamento de doenças." (BRASIL, 2001) 
O Processo desde a captação até a utilização do sangue e/ou derivados é executado e regulado pelo hemocentro estadual que deve estar constantemente monitorado e fiscalizado pela Agência Nacional de Vigilância Sanitária (ANVISA).

A execução da Política Nacional de Sangue segue princípios e diretrizes definidos nos quais institui que a doação de sangue deve ser voluntária e não remunerada; proíbe a comercialização da coleta, processamento, estocagem, distribuição e transfusão do sangue, componentes e hemoderivados; protege a saúde do doador e o receptor através de informações dos procedimentos a serem realizados; obrigatoriedade de responsabilidade, supervisão e assistência médica na triagem de doadores; direito a informação sobre a origem e procedência do sangue; obrigatoriedade de uso de matérias descartáveis que entrarão em contato com o sangue coletado; obrigatoriedade de testagem de cada amostra coletada; participação na fiscalização, vigilância, e controle por entidades civis, permissão de remuneração dos custos dos insumos, reagentes, materiais descartáveis e da mão-de-obra especializada (BRASIL, 2001).

O saneamento básico está intimamente ligado a questão da saúde pública, tanto no que tange a promoção a saúde quanto na prevenção de doenças. Podemos dizer que o saneamento orientado para a Promoção da Saúde abrange a implantação de uma estrutura física composta por sistemas de engenharia, caracterizando o saneamento constitui uma intervenção de engenharia que ocorre no ambiente considerado como espaço físico, voltada para obstaculizar a transmissão de doenças e assegurar a salubridade ambiental, e que compreende a saúde como ausência de doenças. (SOUZA; FREITAS, 2010).

A Fundação Nacional de Saúde a (Funasa), está ligada a implementação do SUS e promove o fortalecimento das instituições estaduais e municipais utilizando recursos que desoneram as tarifas dos serviços e aceleram a universalização do atendimento dos serviços. A Funasa atualmente promove o fomento às soluções de saneamento para prevenção e controle de doenças.

A secretaria de Vigilância em Saúde é a atual responsável pelas ações de vigilância, prevenção e controle de doenças transmissíveis, vigilância de fatores de risco para o desenvolvimento de doenças crônicas não transmissíveis, saúde ambiental e do trabalhador segundo o Ministério da Saúde. A Vigilância em Saúde Ambiental (VSA) atua no controle de mudanças ambientais que interfiram na saúde humana, a fim de identificar medidas de prevenção e controle de riscos ambientais relacionados a doenças ou a outros agravos a saúde. 


\begin{abstract}
Ao restabelecer os processos perdidos do ecossistema, essas soluções da natureza e baseadas na natureza podem potencialmente reconstruir barreiras aos organismos de doenças que se transferem dos animais para os seres humanos. Além disso, essas formas de regeneração das funções do ecossistema poderiam, se combinadas com o gerenciamento sustentável das atividades e infraestrutura humanas, melhorar benefícios como o acesso a suprimentos adequados de água limpa, que têm papéis significativos a desempenhar no gerenciamento de surtos de doenças (EVERARD; JOHNSTON; SANTILLO; STADDON, 2020)
\end{abstract}

A Política de promoção da saúde descreve a promoção do desenvolvimento sustentável, incluindo nesta, a reorientação das práticas de saúde, de modo a permitir a interação: saúde, meio ambiente e desenvolvimento sustentável (BRASIL, 2010b). A saúde é definida pela Organização Mundial de Saúde (OMS) como um completo estado de bem-estar físico, mental e social e não meramente como ausência de doença (SANTOS, 2016).

A política de saúde no Brasil, que deu origem ao SUS, concretamente, consistiu num esforço histórico de construção de uma cidadania focada na perspectiva do reconhecimento do ser social como sujeito de direitos (SACRAMENTO, 2016). E a compreensão da determinação social da saúde na particularidade de um país periférico de desenvolvimento desigual como eixo problematizador e estruturante do novo sistema de saúde a ser construído (PAIM, 2007).

É bem estabelecido que o desenvolvimento regional envolve uma série de fatores que envolve não só o contexto econômico, havendo a necessidade de desenvolver Políticas que, por exemplo, busquem elevar o IDH local, tendem a melhorar a situação geral da população e reduzir, de forma significativa, as despesas públicas locais. Reflexo do cenário global em que o fortalecimento regional passa a afetar diretamente o desenvolvimento das potencialidades locais, determinando, em grande parte, a inserção competitiva do país no mercado global (GADELHA, 2007).

Neste contexto, é notória a importância da relação de desenvolvimento regional com a saúde, visto que diversos fatores podem afetar a saúde humana. A aproximação da temática do desenvolvimento regional com o movimento de promoção da saúde permite a ampliação na compreensão do processo saúde-doença (SANTOS, 2016).

\title{
6 CONCLUSÃo
}

O Sistema Único de Saúde brasileiro (SUS) está completando neste ano de 2020, 30 anos de existência, a partir da sua criação pela lei no 8.080 de 19 de setembro de 1990. Dentre os maiores sistemas de saúde pública do mundo, ele oferece serviços que abrangem categorias das mais simples como um atendimento ambulatorial para medição de glicose, por exemplo, 
até as categorias mais complexas, como o transplante de órgãos. No rol das atividades praticadas pelo SUS podemos também atividades ligadas vigilância sanitária, vigilância epidemiológica, saúde do trabalhador, assistência terapêutica integral, inclusive farmacêutica, a ordenação da formação de recursos humanos na área de saúde, vigilância nutricional e a orientação alimentar, a colaboração na proteção ao meio ambiente, nele compreendido o do trabalho, formulação da política de medicamentos, equipamentos e imunobiológicos, o controle e fiscalização de serviços, produtos e substâncias de interesse para a saúde, a fiscalização e a inspeção de alimentos, água e bebidas para consumo humano, a participação no controle e na fiscalização da produção, transporte e guarda e utilização de substâncias e produtos psicoativos, tóxicos e radioativos, o incremento, em sua área de atuação, do desenvolvimento científico e tecnológico e a formulação e execução da política de sangue e seus derivados.

Em meio a uma pandemia onde a vacinação seria vista como uma ferramenta vital para a sua contensão, mesmo quando quase a totalidade dos entrevistados diz se vacinar em postos de saúde, cerca de 98,8\%, temos ainda $35,7 \%$ dos entrevistados que acreditam não buscar atendimento pelo SUS, o que evidencia a falta de conhecimento por esta parcela dos entrevistados de que a vacinação é prestada pelo SUS.

Apesar de ser tão abrangente e importante para a saúde da população, podemos verificar que o nível de conhecimento de suas áreas atuação é precário, tanto pela população em geral, quanto pelos trabalhadores da área da saúde, tendo em vista que eles deveriam conhecer a fundo o sistema de saúde. A falta de conhecimento sobre as ações do SUS, implica de alguma forma na falta de conhecimento de onde buscar um serviço ou atendimento específico, tendo em vista que se não se sabe o responsável por determinado serviço, se torna complexa a solicitação e cobrança por ele. Também foi evidenciado que o conhecimento acerca do campo de atuação do SUS, bem como, as suas ações podem contribuir diretamente no Desenvolvimento Regional. 


\section{REFERÊNCIAS}

AGUIAR, Z. N. SUS: Sistema Único de Saúde - antecedentes, percurso, perspectiva e desafios. São Paulo SP: Martinari, 2015. 189 p.

AMARAL, V. F.; CAVALCANTE, A. S. P.; FARIAS, Q. L. T.; RIBEIRO, M. A.; ARAÚJO, J. D. G.; GOMES, D. F. Mobilizando estudantes em defesa do Sistema Único de Saúde (SUS): experiências interprofissionais do ver-SUS - Sobral, CE, Brasil. Interface Comunicação, Saúde, Educação, Botucatu, v. 22, n. 2, p. 1787-1797, 2018. Disponível em: http://dx.doi.org/10.1590/1807-57622017.0715. Acesso em: 26 fev. 2021.

ANVISA. Agência Nacional de Vigilância sanitária. Acompanhamento dos temas serviços de interesse para a saúde. Disponível em: http://portal.anvisa.gov.br/20172020/servicos-de-interesse. Acesso em: 01 jul. 2020.

BATAEIRO, M. O. Segurança química e vigilância sanitária de acidentes com produtos perigosos no estado de São Paulo. Boletim Epidemiológico Paulista, São Paulo, v. 13 n. 153-154, p. 69-75, set./out., 2016.

BERMUDEZ, J. A.; ESHER, A.; OSORIO-DE-CASTRO, C. G.; VASCONCELOS, D. M.; CHAVES, C. G.; OLIVEIRA, M. A. et al. Assistência Farmacêutica nos 30 anos do SUS na perspectiva da integralidade. Ciência \& Saúde Coletiva, [s. 1.], v. 23, n.6, p.1937-1951, 2018.

BORTOLINI, G. A.; OLIVEIRA, T. F. V.; SILVA, S. A.; SANTIN, R. C.; MEDEIROS, O. L.; SPANIOL, et al. Ações de alimentação e nutrição na atenção primária à saúde no Brasil. Revista Panamericana de Salud Pública, [s.1.], v. 44, p. 1-8, abr. 2020.

BRASIL. Ministério da Saúde. Lei 8080 de 19 de setembro de 1990. Dispõe sobre as condições para promoção, proteção e recuperação da saúde, a organização e o funcionamento dos serviços correspondentes e dá outras providências. Diário oficial da União. Brasília, 19 set. 1990.

BRASIL. Ministério da Saúde. Lei 8142 de 28 de dezembro de 1990. Dispõe sobre a participação da comunidade na gestão do sistema Único de Saúde (Sus) e sobre as transferências intergovernamentais de recursos financeiros na área da saúde e dá outras providências. Diário oficial da União. Brasília, 28 dez. 1990.

BRASIL. Ministério da Saúde. Vigilância alimentar e nutricional nos serviços de saúde. https://www.saude.gov.br/sgtes. Acesso em: 29 jun. 2020.

BRASIL. Ministério da Saúde. Vigilância em saúde ambiental. Disponível em: https://www.saude.gov.br/vigilancia-em-saude/vigilancia-ambiental. Acesso em: 29 jun. 2020.

BRASIL. Ministério da Saúde. Vacinação, aquisição de imunobiológicos. Disponível em: https://www.saude.gov.br/saude-de-a-z/vacinacao/aquisicao-imunobiologicos. Acesso em: 01 jul. 2020.

BRASIL. Lei no 10205 de 21 de março de 2001. Regulamenta o § 4o do art. 199 da Constituição Federal, relativo à coleta, processamento, estocagem, distribuição e aplicação do 
sangue, seus componentes e derivados, estabelece o ordenamento institucional indispensável à execução adequada dessas atividades, e dá outras providências. Disponível em: http://www.planalto.gov.br/ccivil_03/leis/leis_2001/110205.htm. Acesso em: 01 jul. 2020.

BRASIL. Política Nacional de Desenvolvimento Regional (PNDR), atualizada pelo Decreto $\mathrm{n}^{\circ} 9.810$, de 2019

COSTA, E. A.; ROZENFELD. S. Constituição da Vigilância Sanitária no Brasil. In: ROZENFELD, S. Fundamentos da Vigilância Sanitária. Rio de Janeiro. Editora: Fiocruz, 2000, p. 15-41.

CRUZ, P. J.; VIEIRA, S. C.; MASSA, N. M.; ARAÚJO, T. A.; VASCONCELOS, A. C. Desafios para a Participação Popular em Saúde: reflexões a partir da educação popular na construção de conselho local de saúde em comunidades de João Pessoa. Saúde Soc., São Paulo, v.21, n.4, p.1087-1100, 2012.

EVERARD, M.; JOHHSTON, P.; SANTILLO, D.; STADDON, C. The role of ecosystems in mitigation and management of Covid-19 and other zoonoses. Environmental Science and Policy [s.1.], v.111, p.7-17, 2020.

FIOCRUZ. Pense SUS, inovação. Disponível em: https://pensesus.fiocruz.br/inovacao. Acesso em: 01 jul. 2020.

GADELHA, C. A.; COSTA, L. Integração de fronteiras: a saúde no contexto de uma política nacional de desenvolvimento. Cad. Saúde Pública, Rio de Janeiro, v. 23, supl. 2, p.214-226, 2007. Disponível em: https://doi.org/10.1590/S0102-311X2007001400011. Acesso em: 10 jul. 2020.

HENRIQUETA, T. S.; GENTILLI, R. M. Mundialização do capital e política de saúde: desafios para as práticas integrativas e complementares no SUS. Revista de Políticas Públicas, São Luís, v. 20, n 1, p.103-120, jan./jun., 2016.

LUCCHESE, G.; SETA, M. H.; PEPE, V. L.; OLIVEIRA, G. O. Gestão e Vigilância Sanitária: modos atuais do pensar e fazer. Rio de Janeiro: Editora Fiocruz, 2006, 284p.

PAIM, J. S. Reforma sanitária brasileira: contribuição para compreensão e crítica. 2007, 300 f. Tese (Doutorado em Saúde Coletiva) - Instituto de Saúde Coletiva, Universidade Federal da Bahia, Salvador, 2007.

PAIVA, A. S.; COSTA, M. D. H. Ataques à política de saúde em tempos de crise do capital. Revista de Políticas Públicas, São Luís, v. 20, n 1, p.51-68, jan./jun., 2016. Disponível em: http://dx.doi.org/10.18764/2178-2865.v20n1p51-68. Acesso em: 09 jul. 2020.

SACRAMENTO, H. T.; GENTILLI, R. M. L. Mundialização do capital e política de saúde: desafios para as práticas integrativas e complementares no SUS. Revista de Políticas Públicas, São Luís, v. 20, n 1, p.103-120, jan./jun., 2016. Disponível em: http://dx.doi.org/10.18764/2178-2865.v20n1p103-120. Acesso em: 09 jul. 2020.

SANTOS, D. A.; AZEVEDO, P. V.; AZEVEDO, J. V.; ALVES, T. L.; SOUZA, A. Percorrendo os caminhos da relação entre as políticas públicas de saúde e do meio ambiente. Revista de Políticas Públicas, São Luís, v. 20, n 1, p.103-120, jan./jun., 2016. 
SÃO PAULO. P. M. Prefeitura Municipal de São Paulo. Vigilância de produtos de interesse da saúde. Disponível em:

https://www.prefeitura.sp.gov.br/cidade/secretarias/saude/vigilancia_em_saude/vigilancia_san itaria/index.php?p=222525\#: :text=A\%20Vigil\%C3\%A2ncia\%20de\%20Produtos\%20de,fisc aliza\%C3\%A7\%C3\%A3o\%2C\%20normatiza\%C3\%A7\%C3\%A3o\%2C\%20entre\%20outras . Acesso em: 27 fev. 2021.

SOUZA, C. M.; FREITAS, C. M. A produção científica sobre saneamento: uma análise na perspectiva da promoção da saúde e da prevenção de doenças. Engenharia Sanitária e Ambiental, [s.1.], v. 15, n. 1, p. 65-74, mar. 2010.

TEIXEIRA, J.; CRUZ, C. A.; AZEVEDO, A.P. Universalidade do Atendimento à Saúde no Brasil: impasses e perspectivas. Revista de Políticas Públicas, [s. 1.], v. 20, n. 1, p. 201-220, 2016.

VINUTO, J. Amostragem em Bola de Neve na Pesquisa Qualitativa: um Debate em Aberto. Temáticas, Campinas, v. 22, n. 44, p. 203-220, ago./dez. 2014. 